Ciência Florestal, Santa Maria, v. 24, n. 3, p. 645-653, jul.-set., 2014

ISSN 0103-9954

\title{
TAMANHO E FORMAS DE PARCELAS PARA INVENTÁRIOS FLORESTAIS DE ESPÉCIES ARBÓREAS NA AMAZÔNIA CENTRAL
}

\author{
SIZE OF PLOTS AND FORMS FOR FOREST INVENTORY OF TREE SPECIES IN CENTRAL \\ AMAZON
}

Milena Marmentini de Oliveira ${ }^{1}$ Niro Higuchi ${ }^{2}$ Carlos Henrique Celes ${ }^{3}$ Francisco Gasparetto Higuchi ${ }^{4}$

\section{RESUMO}

Uma das principais ferramentas para quantificar e qualificar o potencial de uma floresta é o inventário florestal. Durante a sua fase de planejamento, é necessário definir a intensidade de amostragem e o tamanho e forma de unidades de amostra para minimizar os custos de coleta de dados, aumentando a precisão do inventário florestal. Este estudo teve como objetivo testar diferentes tamanhos e formas de unidades de amostra para diferentes categorias de diâmetros a altura do peito (DAP). O estudo foi realizado na região de Manaus, incluindo INPA e áreas como a estação experimental da Embrapa em torno da BR-174, no estado do Amazonas, Brasil. Cinco categorias de DAP foram consideradas, como a seguir: 5 centímetros, $10 \mathrm{~cm}, 20 \mathrm{~cm}, 25 \mathrm{~cm}$ e $45 \mathrm{~cm}$. Para todas as categorias de DAP, vinte e três diferentes tamanhos e duas formas foram testados, de $100 \mathrm{~m}^{2}$ a $10.000 \mathrm{~m}^{2}$. O número de amostras foi definido como $\mathrm{n}=30$ para todas as simulações de tamanho, para se confirmar a utilização dos pressupostos do teorema do limite central. A área basal foi utilizada para estimar a incerteza em relação ao verdadeiro valor da média para a comparação entre os diferentes tamanhos para cada categoria de DAP. Quase todos os tamanhos de parcela, em todas as categorias de DAP, obtiveram incertezas inferiores a $10 \%$. Os que obtiveram melhores resultados foram os de $1000 \mathrm{~m}^{2}, 800 \mathrm{~m}^{2}, 1200 \mathrm{~m}^{2}, 2000 \mathrm{~m}^{2}$ e $10.000 \mathrm{~m}^{2}$ respectivamente, para os DAP mínimos de 5, 10, 20, 25 e 45 centímetros. A maioria dos inventários florestais exploratórios no estado do Amazonas tem sido feita para árvores com DAP maior que 10 centímetros, e os tamanhos variando de $1000 \mathrm{a} 2000 \mathrm{~m}^{2}$. Os resultados deste trabalho demonstram que o tamanho para o inventário exploratório é adequado, porém, tamanhos diferentes devem ser usados para DAP maior ou igual a 45 centímetros, que é normalmente o DAP mínimo para os planos de exploração madeireira.

Palavras-chave: levantamentos florestais; suficiência amostral; unidade amostral.

\begin{abstract}
One of the main tools to quantify and to qualify the forest potentials is the forest inventory. During its planning phase it is necessary to define the sampling intensity as well as the sizes and shapes of sample units to minimize data collection costs while increasing the precision of the forest inventory. This study aimed to test different sizes and shapes of sample units for different diameters at breast height (DBH) size categories. The study was carried out in the Manaus region including INPA and EMBRAPA experimental station areas around BR-174 road, in the state of Amazonas, Brazil. Five categories of DBH were considered, as following: above $5 \mathrm{~cm}, 10 \mathrm{~cm}, 20 \mathrm{~cm}, 25 \mathrm{~cm}$ and $45 \mathrm{~cm}$. For all DBH categories, twenty-three different
\end{abstract}

1 . Engenheira Florestal, Msc., Pesquisadora voluntária do Laboratório de Manejo Florestal, Instituto Nacional de Pesquisas da Amazônia, Av. André Araújo, 2936, Petrópolis, CEP 69060-0010, Manaus (AM), Brasil. miflorest@gmail.com

2 Engenheiro Florestal, Pesquisador do Instituto Nacional de Pesquisas da Amazônia, Av. André Araújo, 2936 , Petrópolis, CEP 69067-375, Manaus (AM), Brasil.niro@inpa.gov.br

3 Engenheiro Florestal, Msc., Colaborador do Laboratório de Manejo Florestal, Instituto Nacional de Pesquisas da Amazônia, Av. André Araújo, 2936, Petrópolis, CEP 69060-0010, Manaus (AM), Brasil. carlosceles@yahoo.com

4 Engenheiro Florestal, Msc., Doutorando pelo Programa de Pós-graduação em Engenharia Florestal, Universidade Federal do Paraná, Av. Pref. LothárioMeissner, 900, CEP 80210-170, Curitiba (PR), Brasil. fghiguchi@gmail.com

Recebido para publicação em 9/09/2011 e aceito em 17/04/2013

Ci. Fl., v. 24, n. 3, jul.-set., 2014 
sizes were tested, from $100 \mathrm{~m} 2$ to $10,000 \mathrm{~m} 2$. The number of samples was defined as $\mathrm{n}=30$ for all size simulations to qualify for the use of the central limit theorem assumptions. Basal area was used to estimate uncertainty in relation to the true value of mean for comparison among different sizes for each DBH category. Almost all sample plot sizes in all categories of DAP obtained uncertainties below $10 \%$. The best sample sizes were $1,000 \mathrm{~m}^{2}, 800 \mathrm{~m}^{2}, 1,200 \mathrm{~m}^{2}, 2,000 \mathrm{~m}^{2}$ and $10,000 \mathrm{~m}^{2}$, respectively for minimum DBH of 5 , $10,20,25$ and $45 \mathrm{~cm}$. Most of exploratory forest inventories in the state of Amazonas have been done for trees with DBH greater than $10 \mathrm{~cm}$, and sizes have varied from 1,000 to 2,000 $\mathrm{m} 2$. The findings of this work demonstrated that the size for exploratory inventory is adequate. However, different size should be used for $\mathrm{DBH}$ greater than $45 \mathrm{~cm}$, which is usually the minimum DBH for logging plans.

Keywords: forest surveys; sample sufficiency; sampling unit.

\section{INTRODUÇÃO}

A Amazônia brasileira tem uma grande riqueza florística, mas os representantes dessa flora e a sua distribuição ainda são pouco conhecidos (FEARNSIDE, 2006). Para ter um conhecimento correto da Amazônia é preciso estudos de quantificação e qualificação da vegetação existente. Para tanto, utiliza-se como ferramenta de estudo, o inventário florestal.

O primeiro inventário florestal realizado na Amazônia brasileira ocorreu em 1948, com o intuito de saber o potencial madeireiro para carvoaria (OLIVEIRA, 2000). O histórico de ocupação da Amazônia sempre foi caracterizado para fins de exploração da matéria-prima existente, no caso, a madeira de algumas espécies de potencial econômico. Nas últimas décadas, com o aumento da preocupação do papel das florestas nas mudanças climáticas globais, aumentou o interesse de pesquisadores e do governo em quantificar o volume de madeira e carbono para saber o potencial da floresta como fonte de equilíbrio para o meio ambiente.

Em geral, a maioria dos inventários realizados na Amazônia visa, principalmente, à estimativa de volume para avaliar a viabilidade técnica e econômica de planos de manejo florestal em terras privadas. Para isso, são inventariados apenas os indivíduos arbóreos com diâmetro a altura do peito (DAP) maior ou igual $25 \mathrm{~cm}$ (na maioria das vezes, maior do que $45 \mathrm{~cm}$ ) e, normalmente, são adotadas equações de volume inadequadas e a identificação das espécies florestais é também bastante precária.

Geralmente o tamanho e forma de parcelas têm sido escolhidos mais pela praticidade e operacionalidade na sua instalação, medição e localização do que qualquer outro motivo
(PÉLLICO NETTO e BRENA, 1997).

Silva (1980) testou a eficiência relativa entre parcelas quadradas, retangulares e circulares na região do Baixo Tapajós - PA, que foi avaliada em relação ao tempo total necessário para estabelecer e medir as unidades amostrais. Parcelas retangulares de $10 \mathrm{~m}$ de largura mostraram-se menos eficientes que as circulares, enquanto que as retangulares de $20 \mathrm{~m}$ foram até $140 \%$ mais eficientes que o mesmo tamanho da forma circular. Contudo, se tamanhos maiores que $2500 \mathrm{~m}^{2}$ tivessem sido testados, provavelmente a eficiência cairia, pois as dimensões da parcela dificultariam o controle das árvores limítrofes, aumentando o tempo. Além disso, segundo Silva (1980), as amostras retangulares de $20 \mathrm{~m}$ de largura foram superiores às de $10 \mathrm{~m}$, sendo o tamanho de $1000 \mathrm{~m}^{2}, 311 \%$ mais eficiente.

Em contrapartida, Mesavage e Grosenbaugh (1956), citado por Ubialli et al. (2008); Bonetes (2003) e Silva (1980) afirmaram que as estimativas, sem tendência, do volume de madeira de uma floresta, podem ser obtidas sendo empregadas parcelas de qualquer tamanho, porém, sua localização não poderá ser tendenciosa. Mas, para isso, é preciso que exista um intervalo limitado de tamanhos, em que a eficiência da amostragem seja a melhor possível. O melhor tamanho pode ser diferenciado de uma amostragem para outra e vai depender do quanto as árvores estão agrupadas e do custo.

Para a FAO (1974), quanto menores forem as unidades amostrais, maior será a precisão, levando em conta que seja utilizado a mesma intensidade. Porém, cada indivíduo da amostra deve dar uma imagem representativa da floresta.

A incerteza de um inventário florestal pode ser influenciada não só pela intensidade de amostragem, como também pelo tamanho das parcelas amostrais. Em Higuchi et al. (1982), há 
uma revisão compreensiva de diferentes tamanhos utilizados em 8 inventários no Brasil, que variaram de $2500 \mathrm{~m}^{2}$ a $10.000 \mathrm{~m}^{2}$. Em nenhum caso, há uma explicação para o tamanho adotado da parcela. $\mathrm{O}$ inventário utilizado para orientar os concorrentes ao Edital de Concessão Florestal do $1^{\circ}$ lote na Flona Jamari (RO) empregou dois tamanhos diferentes de parcelas: de $10 \mathrm{~m} \times 100 \mathrm{~m}\left(1000 \mathrm{~m}^{2}\right)$ para árvores com DAP $\geq 25 \mathrm{~cm}$ e $10 \mathrm{~m} \times 150 \mathrm{~m}\left(1500 \mathrm{~m}^{2}\right)$ para árvores com DAP $\geq 45 \mathrm{~cm}$ (MMA, 2008).

Para árvores com DAP $\geq 25 \mathrm{~cm}$ de florestas primárias da região de Manaus (AM), Higuchi et al. (1982) concluíram que o tamanho ideal de parcela é de $3000 \mathrm{~m}^{2}$, levando em consideração as análises estatísticas realizadas, as quais apresentaram valores constantes de coeficiente de variação e erro amostral, a partir de parcelas com $3000 \mathrm{~m}^{2}$.

Em Santarém (PA), Queiroz (1977) concluiu que a parcela de $3200 \mathrm{~m}^{2}$ foi suficiente para atingir a precisão desejada. Silva (1980) concluiu, para a mesma área, que o tamanho ideal é de $900 \mathrm{~m}^{2}$ para diâmetro (DAP) entre $15 \mathrm{~cm}$ e 49,99 $\mathrm{cm}$ e para DAP $\geq 45 \mathrm{~cm}$ o ideal é $2500 \mathrm{~m}^{2}$, ambos na forma quadrada. Salienta-se que o maior tamanho de parcela testado foi de $2500 \mathrm{~m}^{2}$.

Cavalcanti et al. (2009) estudaram tamanhos e formas de parcelas para inventários florestais de espécies comerciais da Amazônia. Foram medidos todos os indivíduos arbóreos com DAP $\geq 40 \mathrm{~cm}$ de interesse comercial. A parcela que obteve menor erro foi a de 2 ha ( $50 \mathrm{~m} \times 400 \mathrm{~m})$.

Ogaya (1968), citado por Ubialli et al. (2008), afirmou que a aplicação de unidades amostrais de pequenas dimensões onera os custos do inventário em virtude do aumento no comprimento dessas parcelas para cobrir a mesma superfície levantada. Afirmou, em consequência, que a abertura de picadas e os deslocamentos de pessoal representam acréscimo nos custos dos levantamentos em florestas tropicais. Por essa razão e considerando que a escolha do tamanho da parcela é um compromisso entre a teoria estatística, as condições práticas mais favoráveis de trabalho e os custos, recomendou o emprego de parcelas retangulares com $20 \mathrm{~m}$ de largura e de 100 a $125 \mathrm{~m}$ de comprimento.

Um dos grandes problemas nos inventários realizados na floresta amazônica é a grande variabilidade de metodologias empregadas, principalmente no que se refere a tamanho e forma de parcela e a definição do diâmetro mínimo de inclusão. Oliveira (2000) avaliando diferentes inventários quantitativos em florestas de terra firme na Amazônia brasileira teve grandes dificuldades de comparar os resultados devido à grande variabilidade de metodologias empregadas. Segundo esse autor, é necessário utilizar metodologias com maior eficiência e que permitam a extrapolação dos dados para um universo melhor definido e efetivamente maior do que a área amostrada.

Desta forma, este trabalho vem auxiliar na definição do tamanho ideal de parcela para inventários florestais na Amazônia, para cinco categorias de diâmetros (DAP) mínimos, com o mesmo número de unidades de amostra para cada categoria, levando em consideração a precisão e o custo.

\section{MATERIAL E MÉTODO}

\section{Caracterização da área de estudo}

As áreas de estudo estão localizadas ao longo da rodovia BR-174, que liga a cidade de Manaus à cidade de Boa Vista. Na margem esquerda km 50 da BR-174 está a Estação Experimental de Silvicultura Tropical (EEST) do Instituto Nacional de Pesquisas da Amazônia (INPA). O acesso à EEST dá-se até $\mathrm{okm} 21$ da estrada vicinal ZF-2. Na margem direita, no km 51 da BR-174, encontra-se o Parque Fenológico localizado na Estação Experimental do Distrito Agropecuário da SUFRAMA, pertencente à EMPRAPA Amazônia Ocidental, entre as coordenadas geográficas $2^{\circ} 30^{\prime} 36^{\prime \prime} \mathrm{S}$ e $2^{\circ} 30^{\prime} 42^{\prime \prime} \mathrm{N}$ e $60^{\circ} 01^{\prime} 29^{\prime \prime} \mathrm{W}$ e 60 $01^{\circ} 46^{\prime \prime} \mathrm{E}$. Essas duas áreas distam cerca de $41 \mathrm{~km}$ e possuem as mesmas características físicas e biológicas

O clima dessa região é quente e úmido (BASTOS et al., 1986), apresentando umidade relativa média muito alta, variando de 84 a $90 \%$. A precipitação total anual varia de 2000 a $3000 \mathrm{~mm}$. As médias anuais de temperatura do ar variam de $25,6^{\circ} \mathrm{C}$ a $27,6^{\circ} \mathrm{C}$. O tipo climático é Am na classificação de Köppen.

Ferraz et al. (1998) fizeram levantamento dos solos de platô, encosta e baixio, nas localidades da área de estudo e constataram que os solos dos platôs apresentaram textura argilosa; nas encostas, variaram de argiloarenoso próximos aos platôs a arenoargilosos, próximos aos baixios e, os solos nas áreas de baixio, apresentaram textura arenosa, definindo que, para a EEST-INPA, o solo da região pode ser classificado como Latossolo Amarelo nos platôs; Podzólicos Vermelho-Amarelo nas encostas 
e Arenossolos hidromórficos nos baixios.

Área de "sub-região dos baixos platôs da Amazônia" foi a denominação dada por RADAMBRASIL (1978). Na bacia do Tarumãzinho, área contígua à área de estudo, por meio do inventário florestal de 96 hectares, foram encontrados 14.922 indivíduos com DAP $\geq 25 \mathrm{~cm}$, distribuídos em 51 famílias botânicas diferentes, com 409 espécies para 206 gêneros. As espécies mais abundantes foram castanha-jarana (Lecythidaceae), inharé (Moraceae), ucuúba (Mirysticaceae) e uxi (Humiriaceae), com uniforme distribuição espacial dentro da área (HIGUCHI et al., 1985).

\section{Coleta de dados}

Os dados usados neste estudo foram obtidos a partir dos inventários florestais realizados em parcelas permanentes: duas parcelas de 5 ha, 14 parcelas de 1 ha e uma parcela de 30 ha inventariadas a $100 \%$.

$\mathrm{Na}$ EEST estão alocadas três parcelas permanentes: 2 transectos de 5 ha $(20 \mathrm{~m} \times 2500 \mathrm{~m})$ cada, divididos em subparcelas de $20 \mathrm{~m}$ x $20 \mathrm{~m} \mathrm{e}$ uma parcela de 30 ha $(500 \mathrm{~m}$ x $600 \mathrm{~m})$ dividida em subparcelas de $10 \mathrm{~m}$ x $10 \mathrm{~m}$.

No Parque Fenológico da Estação Experimental do Distrito Agropecuário da SUFRAMA, pertencente à EMPRAPA Amazônia Ocidental estão alocadas as 14 parcelas de um hectare divididas em subparcelas de $10 \mathrm{~m}$ x $100 \mathrm{~m}$.

A amostragem utilizada neste projeto foi a Aleatória Simples, também conhecida como Aleatória Irrestrita. Foram realizadas simulações em cinco categorias de diâmetro a altura do peito (DAP): DAP $\geq 5 \mathrm{~cm} \leq 10$ centímetros, DAP $\geq$ 10 centímetros, DAP $\geq 20$ centímetros, DAP $\geq$ 25 centímetros e DAP $\geq 45$ centímetros. Para todas as categorias de DAP, vinte e três diferentes tamanhos e duas formas foram testados, de $100 \mathrm{~m}^{2}$ a $10.000 \mathrm{~m}^{2}$.

O número de unidades de amostra para cada categoria de tamanho e forma de parcela foi de 30 (n), com 30 repetições para cada simulação. Para a categoria de $\mathrm{DAP} \geq 5 \mathrm{~cm} \leq 10 \mathrm{~cm}$ foram realizadas, no total, 210 simulações (sete dimensões de unidades amostrais multiplicada por 30 repetições). Para as categorias de DAP $\geq 10 \mathrm{~cm}, \mathrm{DAP} \geq 20 \mathrm{~cm}$ e DAP $\geq 25 \mathrm{~cm}$ foram realizadas 420 simulações (14 dimensões de unidades amostrais multiplicadas por 30 repetições) para cada categoria. Já para o DAP $\geq 45 \mathrm{~cm}$ foram obtidas 330 simulações
(11 dimensões de unidades amostrais multiplicada por 30 repetições).

A variável de interesse para este estudo foi a incerteza em porcentagem (\%), segundo IPCC (2006), da área basal por parcela. Como segue fórmula abaixo:

$$
\operatorname{Incertez} a(\%)=\left\{\frac{(z) * \text { erropadrão }}{\bar{x}}\right\} * 100
$$

Em que: $\mathrm{z}=$ constante quando considerado um nível de confiabilidade de $95 \%$.

Foi utilizada a Análise de Variância (ANOVA) de um fator, tendo como variável dependente a incerteza e independente os diferentes níveis de tamanho e forma de parcela. Após resultado da ANOVA foi aplicado teste post hoc Tukey para determinar quais tamanhos e formas que são, de fato, estatisticamente diferentes.

Esta quantidade de amostra (30) e esse número de repetições (30) permitem a utilização dos recursos do teorema de limite central para superar a forma diferente do "sino" da distribuição normal dos dados (WEISS e HASSET, 1982). O objetivo é não violar a condicionante da normalidade para não perder a robustez da estimativa da incerteza.

$\mathrm{Na}$ simulação dos tamanhos e formas de parcelas para a categoria de DAP $\geq 5 \mathrm{~cm} \leq 10 \mathrm{~cm}$ foi utilizada a parcela de 30 ha da EEST. Nas demais categorias, para que todos os tamanhos e formas de parcelas fossem simulados, foi necessário trabalhar com as parcelas de forma contígua, construindo os tamanhos conforme as subparcelas de cada parcela permanente. Como algumas parcelas estão divididas em subparcelas de $10 \mathrm{~m}$ x $10 \mathrm{~m}$ e outras em $20 \mathrm{~m}$ x $20 \mathrm{~m}$, foram considerados quatro áreas diferentes denominadas (i) área 1 - parcela de 30 ha; (ii) área 2 - parcela de 30 ha + os dois transectos (40 ha); (iii) área 3 - parcela de 30 ha + quatorze parcelas da EMBRAPA (44,4 ha) e (iv) área 4 - parcela de 30 ha + os dois transectos + quatorze parcelas da EMBRAPA (53,28 ha).

Na Tabela 1 são apresentadas as categorias de DAP mínimos, as dimensões das parcelas testadas e as áreas em que foram testadas. 
TABELA 1: Categorias de diâmetros (DAP) mínimos, tamanhos e formas das parcelas dentro de cada área e dentro de cada categoria de DAP mínimo.

TABLE 1: Categories of minimum (DBH) diameters, sizes and shapes of the plots within each area and within each category of minimum DBH.

\begin{tabular}{ccccc}
\hline Categorias de DAP mínimos & Área 1 & Área 2 & Área 3 & Área 4 \\
\hline & $10 \mathrm{~m} \times 10 \mathrm{~m}$ & - & - & - \\
& $10 \mathrm{~m} \times 20 \mathrm{~m}$ & - & - & - \\
& $10 \mathrm{~m} \times 50 \mathrm{~m}$ & - & - & - \\
$\mathrm{DAP} \geq 5 \leq 10 \mathrm{~cm}$ & $10 \mathrm{~m} \times 100 \mathrm{~m}$ & - & - & - \\
& $20 \mathrm{~m} \times 20 \mathrm{~m}$ & - & - & - \\
& $20 \mathrm{~m} \times 50 \mathrm{~m}$ & - & - & - \\
\hline $\mathrm{DAP} \geq 10 \mathrm{~cm}$ & $20 \mathrm{~m} \times 100 \mathrm{~m}$ & - & & \\
$\mathrm{DAP} \geq 20 \mathrm{~cm}$ & $10 \mathrm{~m} \times 50 \mathrm{~m}$ & $20 \mathrm{~m} \times 40 \mathrm{~m}$ & $10 \mathrm{~m} \times 100 \mathrm{~m}$ & $20 \mathrm{~m} \times 100 \mathrm{~m}$ \\
$\mathrm{DAP} \geq 25 \mathrm{~cm}$ & $20 \mathrm{~m} \times 50 \mathrm{~m}$ & $20 \mathrm{~m} \times 60 \mathrm{~m}$ & $10 \mathrm{~m} \times 200 \mathrm{~m}$ & $20 \mathrm{~m} \times 200 \mathrm{~m}$ \\
& $50 \mathrm{~m} \times 50 \mathrm{~m}$ & $40 \mathrm{~m} \times 40 \mathrm{~m}$ & $50 \mathrm{~m} \times 100 \mathrm{~m}$ & $20 \mathrm{~m} \times 260 \mathrm{~m}$ \\
& - & $60 \mathrm{~m} \times 60 \mathrm{~m}$ & $50 \mathrm{~m} \times 200 \mathrm{~m}$ & - \\
& - & $100 \mathrm{~m} \times 100 \mathrm{~m}$ & - & - \\
DAP $\geq 45 \mathrm{~cm}$ & - & $20 \mathrm{~m} \times 60 \mathrm{~m}$ & $10 \mathrm{~m} \times 100 \mathrm{~m}$ & $20 \mathrm{~m} \times 100 \mathrm{~m}$ \\
& - & $40 \mathrm{~m} \times 40 \mathrm{~m}$ & $10 \mathrm{~m} \times 200 \mathrm{~m}$ & $20 \mathrm{~m} \times 200 \mathrm{~m}$ \\
& - & $60 \mathrm{~m} \times 60 \mathrm{~m}$ & $50 \mathrm{~m} \times 100 \mathrm{~m}$ & $20 \mathrm{~m} \times 260 \mathrm{~m}$ \\
& - & $100 \mathrm{~m} \times 100 \mathrm{~m}$ & $50 \mathrm{~m} \times 200 \mathrm{~m}$ & - \\
\hline
\end{tabular}

\section{RESULTADOS E DISCUSSÃO}

Para cada categoria de DAP mínimo e para cada tamanho e forma de parcelas, foram calculadas as médias das incertezas. Segundo Péllico Netto e Brena (1997), o máximo de erro aceitável nos inventários florestais é de 10\%. Quando se pensa em incerteza, considerando $\mathrm{z}=1,96$ esse valor praticamente dobra, para $20 \%$, devido ao conceito da mesma. Para este trabalho optou-se por admitir uma incerteza de no máximo $10 \%$, tornando a análise mais criteriosa.

Na Tabela 2 são apresentados os valores das médias das incertezas de cada tamanho de parcela dentro de cada categoria de DAP mínimo. Os valores acima de 10\% não fizeram parte da análise de variância. Foram considerados para a ANOVA apenas os valores abaixo de 10\%. Quando houve diferença significativa entre os tamanhos de parcelas $(\mathrm{p}<0,05)$ foi realizado o teste de Tukey para verificação de quais parcelas se diferenciavam entre si.

$\mathrm{Na}$ categoria de DAP $\geq 5 \mathrm{~cm} \leq 10 \mathrm{~cm}$ foram observadas 3 dimensões de parcelas, sendo duas delas de mesmo tamanho, com a incerteza abaixo de $10 \%$. O tamanho $10 \mathrm{~m} \times 100 \mathrm{~m}$, com a incerteza de $8,2 \%$, o tamanho $20 \mathrm{~m} \times 50 \mathrm{~m}$ com a incerteza de $7,3 \%$ e o tamanho de $20 \mathrm{~m}$ x $100 \mathrm{~m}$ com a incerteza de $5,3 \%$.

De acordo com o teste post hoc de Tukey, esses mesmos 3 tamanhos não obtiveram diferença significativa, não sendo diferentes entre si, podendo-se afirmar que qualquer uma dessas três parcelas trará resultados confiáveis para inventários de DAP $\geq 5 \mathrm{~cm}$. Quando se leva em consideração a minimização de custos, utiliza-se a menor parcela, ou seja, a de $1000 \mathrm{~m}^{2}$. Conforme Silva (1980), que conclui que, para parcelas retangulares, a largura de $20 \mathrm{~m}$ tem resultados mais satisfatórios, a parcela de $20 \mathrm{~m}$ x $50 \mathrm{~m}$ é a mais coerente, seguindo o objetivo desta pesquisa.

$\mathrm{Na}$ segunda categoria de DAP $\geq 10 \mathrm{~cm}$, apenas um tamanho de parcela obteve o valor de incerteza maior que $10 \%, 10 \mathrm{~m}$ x $50 \mathrm{~m}$ com uma incerteza de $12,6 \%$, os demais tamanhos e formas de parcelas obtiveram incertezas abaixo da tolerável estabelecida.

Perante o resultado do teste de Tukey, o 
TABELA 2: Valores de incerteza em porcentagem dos tamanhos de parcelas dentro de cada categoria de DAP mínimo.

TABLE 2: Uncertainty values in percentage of plot size within each category of minimum DBH.

\begin{tabular}{|c|c|c|c|c|c|c|}
\hline \multirow{2}{*}{$\begin{array}{c}\text { Dimensão } \\
\text { das Unidades } \\
\text { Amostrais }\end{array}$} & \multirow{2}{*}{$\begin{array}{l}\text { Área das } \\
\text { Unidades } \\
\text { Amostrais }\end{array}$} & \multicolumn{5}{|c|}{ Incerteza $(\%)$} \\
\hline & & $\mathrm{DAP} \geq 5 \leq 10 \mathrm{~cm}$ & $\mathrm{DAP} \geq 10 \mathrm{~cm}$ & $\mathrm{DAP} \geq 20 \mathrm{~cm}$ & $\mathrm{DAP} \geq 25 \mathrm{~cm}$ & $\mathrm{DAP} \geq 45 \mathrm{~cm}$ \\
\hline $10 \mathrm{~m} \times 10 \mathrm{~m}$ & $100 \mathrm{~m}^{2}$ & $23,69^{1}$ & - & - & - & - \\
\hline $10 \mathrm{~m} \times 20 \mathrm{~m}$ & $200 \mathrm{~m}^{2}$ & $16,86^{1}$ & - & - & - & - \\
\hline $20 \mathrm{~m} \times 20 \mathrm{~m}$ & $400 \mathrm{~m}^{2}$ & $11,25^{1}$ & - & - & - & - \\
\hline $10 \mathrm{~m} \times 50 \mathrm{~m}$ & $500 \mathrm{~m}^{2}$ & $11,87^{1}$ & $12,56^{1}$ & $15,63^{1}$ & $17,57^{1}$ & - \\
\hline $20 \mathrm{~m} \times 40 \mathrm{~m}$ & $800 \mathrm{~m}^{2}$ & & 8,86 & $10,74^{1}$ & $13,39^{1}$ & - \\
\hline $10 \mathrm{~m} \times 100 \mathrm{~m}$ & $1000 \mathrm{~m}^{2}$ & 8,21 & 8,92 & $10,87^{1}$ & $12,06^{1}$ & - \\
\hline $20 \mathrm{~m} \times 50 \mathrm{~m}$ & $1000 \mathrm{~m}^{2}$ & 7,30 & 8,21 & $10,40^{1}$ & $11,31^{1}$ & - \\
\hline $20 \mathrm{~m} \times 60 \mathrm{~m}$ & $1200 \mathrm{~m}^{2}$ & - & 7,31 & 9,07 & $10,66^{1}$ & $20,56^{1}$ \\
\hline $40 \mathrm{~m} \times 40 \mathrm{~m}$ & $800 \mathrm{~m}^{2}$ & - & 6,30 & 8,28 & 8,80 & $16,55^{1}$ \\
\hline $10 \mathrm{~m} \times 200 \mathrm{~m}$ & $2000 \mathrm{~m}^{2}$ & - & 6,46 & 8,31 & 9,31 & $17,84^{1}$ \\
\hline $20 \mathrm{~m} \times 100 \mathrm{~m}$ & $2000 \mathrm{~m}^{2}$ & 5,33 & 6,34 & 7,82 & 8,77 & $17,76^{1}$ \\
\hline $50 \mathrm{~m} \times 50 \mathrm{~m}$ & $1000 \mathrm{~m}^{2}$ & - & 5,35 & 6,66 & 7,81 & $14,55^{1}$ \\
\hline $60 \mathrm{~m} \times 60 \mathrm{~m}$ & $3600 \mathrm{~m}^{2}$ & - & 4,66 & 5,40 & 6,57 & $14,00^{1}$ \\
\hline $20 \mathrm{~m} \times 200 \mathrm{~m}$ & $4000 \mathrm{~m}^{2}$ & - & 4,51 & 5,34 & 6,48 & $12,95^{1}$ \\
\hline $50 \mathrm{~m} \times 100 \mathrm{~m}$ & $5000 \mathrm{~m}^{2}$ & - & 4,03 & 5,01 & 5,85 & $12,39^{1}$ \\
\hline $20 \mathrm{~m} \times 260 \mathrm{~m}$ & $5200 \mathrm{~m}^{2}$ & - & 5,34 & 6,35 & 6,55 & $12,35^{1}$ \\
\hline $50 \mathrm{~m} \times 200 \mathrm{~m}$ & $10.000 \mathrm{~m}^{2}$ & - & 3,12 & 3,78 & 4,64 & 9,53 \\
\hline $100 \mathrm{~m} \times 100 \mathrm{~m}$ & $10.000 \mathrm{~m}^{2}$ & - & 2,99 & 3,58 & 4,32 & 8,35 \\
\hline $20 \mathrm{~m} \times 600 \mathrm{~m}$ & $12.000 \mathrm{~m}^{2}$ & - & - & - & - & 9,21 \\
\hline
\end{tabular}

Em que: ${ }^{1}$ Incertezas acima de $10 \%$.

TABELA 3: Divisão dos tamanhos e formas de parcelas dentro de grupos. Dentro de cada grupo, as parcelas não diferem entre si.

TABLE 3: Division of sizes and shapes of plots within groups. The plots do not differ within each group.

\begin{tabular}{|c|c|c|}
\hline Grupo 1 & Grupo 2 & Grupo 3 \\
\hline $20 \mathrm{~m} \times 40 \mathrm{~m}\left(800 \mathrm{~m}^{2}\right)$ & $20 \mathrm{~m}$ x $60 \mathrm{~m}\left(1200 \mathrm{~m}^{2}\right)$ & $50 \mathrm{~m} \times 100 \mathrm{~m}\left(5000 \mathrm{~m}^{2}\right)$ \\
\hline $10 \mathrm{~m} \times 100 \mathrm{~m}\left(1000 \mathrm{~m}^{2}\right)$ & $40 \mathrm{~m} \times 40 \mathrm{~m}\left(1600 \mathrm{~m}^{2}\right)$ & $50 \mathrm{~m} \times 200 \mathrm{~m}\left(10.000 \mathrm{~m}^{2}\right)$ \\
\hline $20 \mathrm{~m} \times 50 \mathrm{~m}\left(1000 \mathrm{~m}^{2}\right)$ & $10 \mathrm{~m} \times 200 \mathrm{~m}\left(2000 \mathrm{~m}^{2}\right)$ & $100 \mathrm{~m} \times 100 \mathrm{~m}\left(10.000 \mathrm{~m}^{2}\right)$ \\
\hline- & $20 \mathrm{~m} \times 100 \mathrm{~m}\left(2000 \mathrm{~m}^{2}\right)$ & - \\
\hline- & $50 \mathrm{~m} \times 50 \mathrm{~m}\left(2500 \mathrm{~m}^{2}\right)$ & - \\
\hline- & $60 \mathrm{~m} \times 60 \mathrm{~m}\left(3600 \mathrm{~m}^{2}\right.$ & - \\
\hline- & $20 \mathrm{~m} \times 200 \mathrm{~m}\left(4000 \mathrm{~m}^{2}\right)$ & - \\
\hline- & $20 \mathrm{~m} \times 260 \mathrm{~m}\left(5200 \mathrm{~m}^{2}\right)$ & - \\
\hline
\end{tabular}


qual apresentou um número grande de parcelas que diferem entre si e também muitas parcelas que não diferem entre si, foi possível criar três grupos de tamanhos e formas de parcelas conforme Tabela 3. As parcelas dentro de cada grupo não diferem entre si.

Todas as parcelas dos três grupos podem ser usadas com uma boa confiabilidade em inventários florestais de DAP $\geq 10 \mathrm{~cm}$. Para diminuir essa amplitude de variedades de tamanhos e formas de parcelas, optou-se por selecionar uma parcela de cada grupo estabelecido. Essa escolha foi baseada na diminuição dos custos de um inventário florestal e levando em consideração que, para as simulações feitas, em que, foi utilizado um número mínimo de unidades amostrais, igual para cada tamanho de parcela, foi definida a escolha do menor tamanho de parcela e não da menor incerteza. Isso porque não houve diferença significativa dentro de cada grupo. Sendo assim, para o grupo 1 o tamanho escolhido foi o de $20 \mathrm{~m} \mathrm{x} 40 \mathrm{~m}\left(800 \mathrm{~m}^{2}\right)$, para o grupo $2 \mathrm{o}$ tamanho de $20 \mathrm{~m} \times 60 \mathrm{~m}\left(1200 \mathrm{~m}^{2}\right)$ e para o grupo 3 o tamanho de $50 \mathrm{~m} \times 100 \mathrm{~m}\left(5000 \mathrm{~m}^{2}\right)$. A parcela de $800 \mathrm{~m}^{2}$ é a que mais se aplica, pois reduz custo, tempo de implantação e uma incerteza aceitável $(8,86 \%)$, porém, se for pensado em liberação de custos, cabe ao profissional responsável escolher o melhor tamanho para sua atividade.

$\mathrm{Na}$ categoria de DAP $\geq 20 \mathrm{~cm}$, a partir da parcela de $20 \mathrm{~m} \times 60 \mathrm{~m}\left(1200 \mathrm{~m}^{2}\right)$, todas as incertezas deram abaixo do limite estabelecido, ou seja, com um tamanho mínimo de $1200 \mathrm{~m}^{2}$ até o tamanho máximo de $10.000 \mathrm{~m}^{2}$ tem-se uma boa margem de segurança na estimativa de dados de um inventário florestal. No teste de Tukey foi identificado que as diferenças estão entre os tamanhos extremos de parcelas, entre $1200 \mathrm{~m}^{2}$ e $10.000 \mathrm{~m}^{2}$. Desta forma, segundo os objetivos deste estudo, a parcela de $1200 \mathrm{~m}^{2}$ é a mais eficiente, pois com uma intensidade amostral mínima, tem-se uma incerteza dentro do esperado $(9 \%)$.

$\mathrm{Na}$ categoria de DAP $\geq 25 \mathrm{~cm}$, apenas as parcelas com mais de $1600 \mathrm{~m}^{2}$ estão dentro do limite tolerável.

Como ocorreu nas categorias anteriores, podem ser obtidos grupos (Tabela 4) de tamanhos e formas de parcelas, baseados nas suas diferenças significativas das suas incertezas. As parcelas dentro de cada grupo não são diferentes entre si, mas entre os grupos elas se diferem.
TABELA 4: Divisão dos tamanhos e formas de parcelas dentro de grupos. Dentro de cada grupo, as parcelas diferem entre si.

TABLE 4: Division of sizes and shapes of plots within groups. The plots do not differ within each group.

\begin{tabular}{cc}
\hline Grupo 1 & Grupo 2 \\
\hline $40 \mathrm{~m} \times 40 \mathrm{~m}\left(1600 \mathrm{~m}^{2}\right)$ & $20 \mathrm{~m} \times 200 \mathrm{~m}\left(4000 \mathrm{~m}^{2}\right)$ \\
$10 \mathrm{~m} \times 200 \mathrm{~m}\left(2000 \mathrm{~m}^{2}\right)$ & $50 \mathrm{~m} \times 100 \mathrm{~m}\left(5000 \mathrm{~m}^{2}\right)$ \\
$20 \mathrm{~m} \times 100 \mathrm{~m}\left(2000 \mathrm{~m}^{2}\right)$ & $20 \mathrm{~m} \times 260 \mathrm{~m}\left(5200 \mathrm{~m}^{2}\right)$ \\
$50 \mathrm{~m} \times 50 \mathrm{~m}\left(2500 \mathrm{~m}^{2}\right)$ & $50 \mathrm{~m} \times 200 \mathrm{~m}\left(10.000 \mathrm{~m}^{2}\right)$ \\
$60 \mathrm{~m} \times 60 \mathrm{~m}\left(3600 \mathrm{~m}^{2}\right)$ & $100 \mathrm{~m} \times 100 \mathrm{~m}\left(10.000 \mathrm{~m}^{2}\right)$ \\
\hline
\end{tabular}

As parcelas do grupo 1 são as mais coerentes, pois possuem os menores tamanhos. Dentre as deste grupo, destaca-se a parcela de $20 \mathrm{~m}$ x $100 \mathrm{~m}\left(2000 \mathrm{~m}^{2}\right)$, pois é retangular com largura de $20 \mathrm{~m}$. Silva (1980) concluiu ser a mais indicada para levantamentos florestais.

Higuchi et al. (1982) concluíram que, para DAP $\geq 25 \mathrm{~cm}$, a melhor parcela é a de $37,5 \mathrm{~cm} \mathrm{x}$ $150 \mathrm{~cm}\left(5625 \mathrm{~m}^{2}\right)$, porém, parcelas a partir de $3000 \mathrm{~m}^{2}$ possuem pouco ganho de precisão, não justificando o custo/benefício. Comparando os resultados destes autores com o estudo em questão é possível concluir que parcelas entre $2000 \mathrm{~m}^{2} \mathrm{e}$ $3000 \mathrm{~m}^{2}$ são as mais recomendáveis para inventários florestais com DAP $\geq 25 \mathrm{~cm}$.

Para a última categoria de DAP mínimo (DAP $\geq 45 \mathrm{~cm}$ ), foi observado que a grande maioria das parcelas obteve uma incerteza acima de $10 \%$. Apenas as parcelas de $50 \mathrm{~m} \times 200 \mathrm{~m}$ $\left(5000 \mathrm{~m}^{2}\right), 100 \mathrm{~m} \times 100 \mathrm{~m}\left(10.000 \mathrm{~m}^{2}\right)$ e $20 \mathrm{~m} \mathrm{x}$ $600 \mathrm{~m}\left(12.000 \mathrm{~m}^{2}\right)$ atingiram incertezas abaixo do esperado. Como observado em campo, árvores com DAP maiores são, de fato, mais difíceis de serem encontradas, o que justifica o caso de apenas grandes parcelas fornecerem uma boa confiabilidade para um levantamento florestal.

O teste post hoc de Tukey demonstra que as diferenças encontradas estavam entre as parcelas com a incerteza acima de $10 \%$. Nas três parcelas com incertezas abaixo do limite máximo, não foi encontrada diferença significativa. Para inventários florestais com objetivo de exploração madeireira a parcela de $10.000 \mathrm{~m}^{2}$ é a mais adequada. Quanto à forma, fica a critério do profissional responsável em decidir qual a melhor. Na prática, a retangular facilita a instalação. 
Cavalcanti et al. (2009) concluíram que o erro amostral e o coeficiente de variação para DAP $\geq 45 \mathrm{~cm}$ estabilizaram a partir da parcela de $0,75 \mathrm{ha}$, resultado próximo ao encontrado neste estudo. Desta forma, confirma-se que, para esta categoria de DAP mínimo, o tamanho de parcela deve ser de pelo menos 1 ha.

Quanto à forma da parcela, em todas as categorias de diâmetros (DAP mínimo) não foi observada diferença entre o tamanho retangular e o quadrado. Isso pode ter ocorrido devido à dificuldade de se montar parcelas de mesma área amostral, porém, de formas diferentes. Todavia, observando-se a instalação das parcelas em campo, a retangular é mais prática quando comparada com a quadrada, principalmente pelo controle que se tem das laterais da parcela, pelo fato de as retangulares serem menos largas e mais compridas. É observado também que as parcelas retangulares abrangem uma variação maior da floresta em relação à parcela quadrada. Isso se confirma no trabalho de Silva (1980), em que parcelas retangulares de $10 \mathrm{~m}$ de largura mostraram-se menos eficientes que as retangulares de $20 \mathrm{~m}$.

É possível observar na Figura 1 que o comportamento das curvas na variação da incerteza é o mesmo para cada categoria de DAP mínimo. Conforme aumenta o tamanho da parcela, diminui a incerteza, praticamente estabilizando a partir da parcela de $60 \mathrm{~m} \times 60 \mathrm{~m}\left(3600 \mathrm{~m}^{2}\right)$. Apenas na parcela de $20 \mathrm{~m} \mathrm{x} 260 \mathrm{~m}\left(5200 \mathrm{~m}^{2}\right)$ a incerteza aumentou, provavelmente devido ao comprimento ser maior que os demais avaliados. Independentemente do DAP mínimo utilizado em um levantamento florestal existe uma faixa de tamanhos de parcelas passíveis de uso $\left(2000 \mathrm{~m}^{2}\right.$ a $\left.3600 \mathrm{~m}^{2}\right)$, levando em consideração a pequena variação no valor da incerteza (Figura 1).

\section{CONCLUSÕES}

Os tamanhos de parcelas que obtiveram melhores resultados foram $1000 \mathrm{~m}^{2}, 800 \mathrm{~m}^{2}$, $1200 \mathrm{~m}^{2}, 2000 \mathrm{~m}^{2}$ e $10.000 \mathrm{~m}^{2}$ para as categorias de diâmetros (DAP) mínimos de $5 \mathrm{~cm}, 10 \mathrm{~cm}, 20 \mathrm{~cm}$, $25 \mathrm{~cm}$ e $45 \mathrm{~cm}$, respectivamente;

A maioria das parcelas simuladas neste estudo pode ser utilizada nos inventários florestais. Da mesma forma, os inventários que têm sido realizados estão dentro da margem de erro (incerteza) aceitável.

O tamanho de parcela utilizada comumente na Amazônia, pelo Instituto de Pesquisas da Amazônia - INPA, $2500 \mathrm{~m}^{2}$ é de fato um tamanho ótimo de parcela não apenas para a categoria de DAP $\geq 10 \mathrm{~cm}$, mas também para as categorias de DAP mínimos de $20 \mathrm{~cm}$ e $25 \mathrm{~cm}$.

Para as categorias de DAP $\geq 5 \mathrm{~cm} \leq 10 \mathrm{~cm}$ e DAP $\geq 45 \mathrm{~cm}$, buscando garantir uma melhor margem de erro, ou menor incerteza, é necessário que se aumente o tamanho das parcelas, levando em consideração o número de unidades de amostra utilizada.

Os inventários florestais na Amazônia

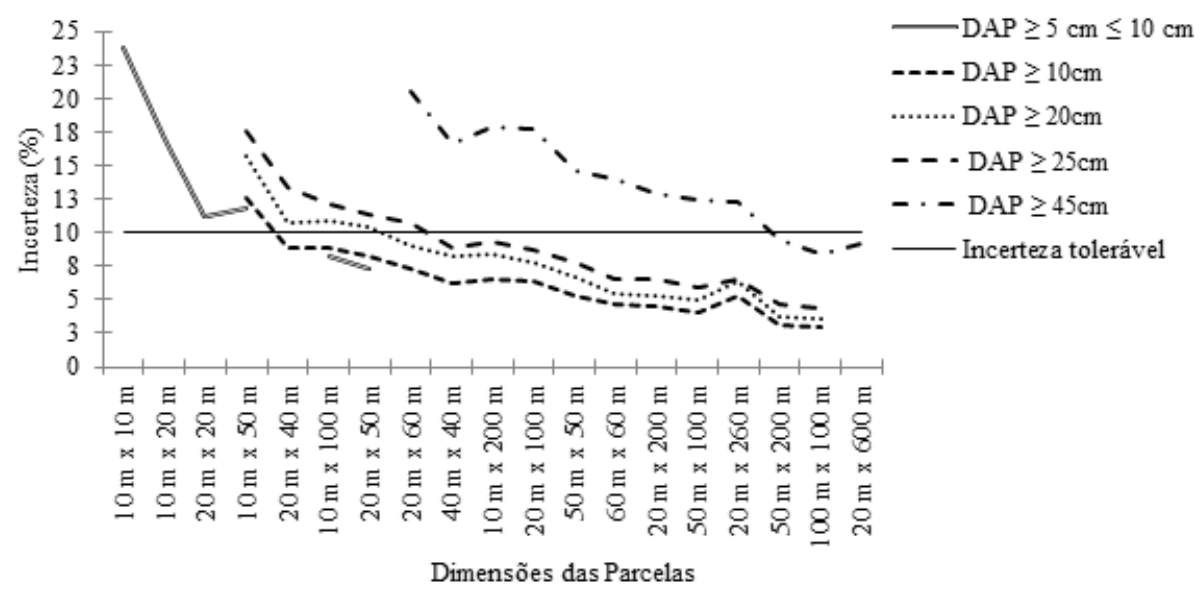

FIGURA 1: Curvas de variação de incerteza de cada tamanho e forma de parcela dentro das categorias mínimas estabelecidas no estudo.

FIGURE 1: Curves of variation of uncertain size and shape of each parcel within the minimum categories set out in the study. 
podem ser realizados com parcelas que variam de $2000 \mathrm{~m}^{2}$ até $3600 \mathrm{~m}^{2}$ com uma boa margem de segurança, para as categorias de DAP mínimo maior ou igual a $10 \mathrm{~cm}, 20 \mathrm{~cm}$ e $25 \mathrm{~cm}$.

\section{REFERÊNCIAS BIBLIOGRÁFICAS}

BASTOS, T. X.et al.The Climate of the Brazilian Amazon region for agricultural purposes; a stateof-the-art. In: Proceedings of the 1st SYMPOSIUM ON THE HUMID TROPICS HELD IN BELÉM, PARÁ. Proceedings... Manaus, 1986.

BONETES, L. Tamanho de parcelas e intensidade amostral para estimar o estoque e índices fitossociológicos em uma floresta ombrófila mista. 2003. 111 f. Dissertação (Mestrado em Ciências Florestais) - Universidade Federal do Paraná, Curitiba, 2003.

CAVALCANTI, F. J. B.et al. Tamanho de unidade de amostra e intensidade amostral para espécies comerciais da Amazônia. Floresta, Curitiba, v. 39, n.1, p. 207-214.2009.

FAO.Manual de inventario florestal com especial referencia a los bosques mistos tropicales. p. 195,Roma. 1974.

FEARNSIDE, P. M. Desmatamento na Amazônia: dinâmica, impactos e controle. Acta Amazonica, Manaus, v. 36, n. 3, p. 395-400,2006.

FERRAZ, J. et al. Pesquisas Florestais para Conservação da Floresta e Reabilitação de Áreas Degradadas da Amazônia: Distribuição dos solos ao longo de dois transectos em floresta primária ao norte de Manaus (AM). Manaus. 1998. 111-143.

HIGUCHI, N.et al. Tamanho de parcela amostral para inventários florestais. Acta Amazonica, Manaus, v. 12, n. 1, p. 91-103.1982

HIGUCHI, N.et al.Devoloping bole wood volume equations for a group of tree species of Central Amazon (Brazil).Commonwealth For. Review, v. 64, n. 1, p. 33-41. 1985.

IPCC - Intergovernmental Panel on Climate Change.2006. IPCC Guidelines for National Greenhouse Gas Inventories. Diponível em: www. ipcc.ch. Acesso: 04 de junho de 2008.

MMA - Ministério do Meio Ambiente.Resultados do Inventário Florestal da Floresta Nacional do Jamari. Disponível em:

$\mathrm{http}: / / \mathrm{mma}$.gov.br/estruturas/sfb/_arquivos/flona jamari_final_anexo06_resultados_do_inventario_ florestal.pdf. Acesso em 25 de janeiro de 2008.

MESAVAGE， C.; GROSENBAUGH， L. R. Efficiency of Several cruising designs on small tracts in North Arkansas. Journal of Forestry, Washington, v. 3 n. 9, p. 569-76, 1956.

OGAYA, N. Kubierungsformeln und Bestandesmassenformeln.Inaugural- Dissertation .Univ., Nat.-Math.Fak, Freiburg, 1968. 85 p.

OLIVEIRA, A. A. Inventários quantitativos de árvores em matas de terra firme: histórico com enfoque na Amazônia brasileira. Acta Amazonica, Manaus, v. 30, n.4, p. 543-567, 2000.

PÉLLICO NETTO, S.; BRENA, D. A. Inventário Florestal. Editorado pelos autores. v. 1, 1997.316 p. QUEIROZ, W. T. Efeitos da variação estrutural em unidade amostral na aplicação do processo de amostragem em conglomerados nas florestas do Planalto do Tapajós. Floresta, v. 8, n. 1, p.19-23,1977.

RADAMBRASIL. Programa de Integração Nacional. Levantamentos de Recursos Naturais. v. 18 (Manaus) - RADAM (projeto) DNPM, Ministério das Minas e Energia. Brasil. 1978. $626 \mathrm{p}$.

SILVA, J. N. M. Eficiência de diversos tamanhos e formas de unidades de amostras aplicadas em inventário florestal na região do Baixo Tapajós. 1980. 84 f. Dissertação (Mestrado em Ciências Florestais) - Universidade Federal do Paraná, Curitiba, 1980.

UBIALLI, J. A.et al. Comparação de métodos e processos de amostragem para estudos fitossociológicos em uma floresta ecotonalna região norte matogrossense. Floresta, Curitiba, v. 39, n. 3, p. $511-523,2008$.

WEISS, N.; HASSET, M. Introductory statistics. Reading: Addison-Wesley.1982. p.651. 